A patient suffering from chronic alcoholic Child B cirrhosis underwent oesophagogastroduodenoscopy because of anemia by means of a Pentax EG-2730. Esophageal varices with cherry red spots were found. Multiband ligation (Saeed MultiBand Ligator MBL-6; Wilson-Cook Medical, Winston-Salem, North Carolina, USA) was carried out. The Opti-Vu barrel with preloaded bands was attached to the tip of the endoscope, with the barrel advanced onto the tip as far as possible. The banding was performed successfully without difficulty. However, the Opti-Vu barrel was then found to be missing from the tip of the endoscope. The endoscope was inserted again, and the barrel was found in the upper end of the esophagus (Figure 1). The barrel was finally retrieved by means of a foreign-body forceps (Figure 2). There was no bleeding or trauma related to this detached barrel or its removal and the patient recovered uneventfully. We found later that the model we used (MBL-6) was suitable for an endoscope with an outer diameter of 9.5-

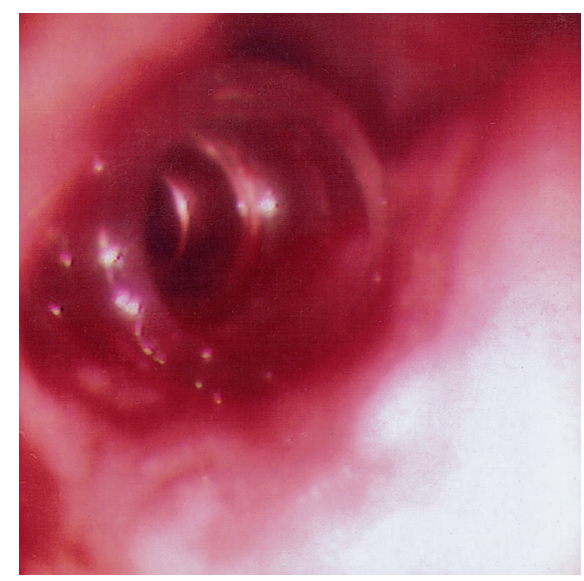

Figure 1 The detached Opti-Vu barrel was found in the upper end of the esophagus on re-introduction of the endoscope.

\title{
A Potentially Serious Disadvantage of Multiband Device Over Conventional Ligation Using an Overtube
}

$13 \mathrm{~mm}$. The endoscope we used, however, had an outer diameter of only $9 \mathrm{~mm}$.

There are very few reports on complications with this new ligator. When this multiple-band ligator is used, the trigger cords that are attached to the transparent tubing are also detached when all six bands are fired. With a conventional band ligator, the string is still firmly attached to the barrel, especially after the band is fired, by further tightening of the trigger cord.

This case reminded us that multiband ligators should be properly checked for compatibility with the endoscope. It is also advisable to check for a firm fit between the barrel and the endoscope. When using multiband ligators, we should pay the same attention to checking whether the barrel is still on the tip of the endoscope after the procedure, as we do to checking the number of gauzes recovered after a laparotomy.

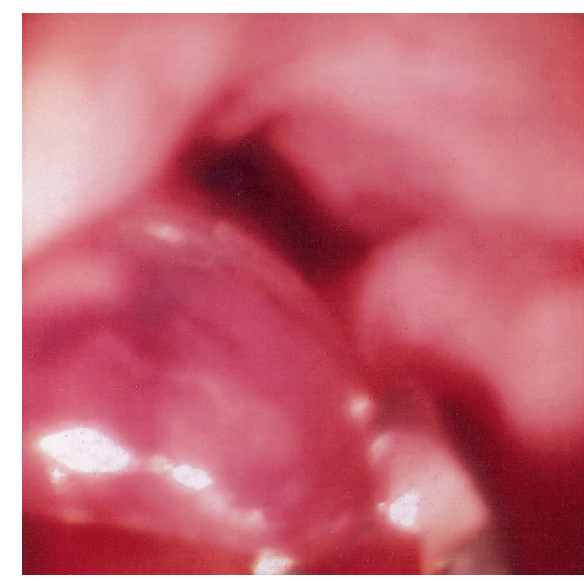

Figure 2 The detached barrel was successfully removed using a foreign-body forceps.

\section{S. Leung, C. W. Chan, W. H. Chow, K. H. Chan, W. K. Kwan \\ Department of Medicine, Yan Chai Hospital, Hong Kong, China}

\section{Corresponding Author}

\section{S. Leung, M.B., Ch.B.}

B14N, Department of Medicine Yan Chai Hospital

Yan Chai Street

Tsuen Wan

New Territories

Hong Kong SAR

China

Fax: $\quad+852-2417-3307$

E-mail: ambroseleung@hotmail.com 